\title{
NEW INSIGHTS INTO SCHIZOPHRENIA: A LOOK AT THE EYE AND RELATED STRUCTURES
}

\author{
Darija Jurišićc ${ }^{1}$, Ivan Ćavar ${ }^{2}$, Antonio Sesar ${ }^{1}$, Irena Sesar ${ }^{1}$, Jakša Vukojević ${ }^{3}$ \& Marko Ć urković ${ }^{3}$ \\ ${ }^{I}$ Department of Ophthalmology, University Clinical Hospital Mostar, Mostar, Bosnia and Herzegovina \\ ${ }^{2}$ Department of Physiology, School of Medicine University of Mostar, Mostar, Bosnia and Herzegovina \\ ${ }^{3}$ University Psychiatric Hospital Vrapče, Zagreb, Croatia
}

\author{
received: 12.3.2019;
}

revised: 9.5.2019;

accepted: 15.5 .2019

\section{SUMMARY}

Background: Schizophrenia is a multifactorial neurodevelopmental disorder associated with cognitive dysfunction and changes in primary sensory processing. This article aims to explore the current insights into the relationship between schizophrenia and different visual disturbances.

Methods: To provide a literature review of visual impairments in schizophrenia, we performed a PubMed/MEDLINE and Scopus search to identify all articles in English on the topic up to the end of 2018.

Results: Multiple retinal functional and structural abnormalities are found in patients with schizophrenia. Wider retinal venules suggest chronically insufficient brain supply of oxygen and this could contribute to the occurrence of psychotic symptoms. Optical coherence tomography studies showed that retinal nerve fiber layer, macular thickness, and macular volume were significantly lowered in the chronic phase of schizophrenia. Results from electroretinogram recordings have demonstrated different declinations such as abnormalities of a - wave activity in the photoreceptors or b-wave activity in the bipolar and Muller cells. Abnormalities in eye movements, such as a notable decrease in saccades and smooth pursuit eye movements, are one of the most reliable and reproducible impairments associated with schizophrenia. Disrupted visual processing of the magnocellular pathway may result in a decrease of contrast sensitivity, sensory processing, orientation discrimination, visual integration, trajectory and spatial localization, backward masking and motion tracking. Visual perceptual abnormalities occur in more than $60 \%$ of schizophrenic patients and these are visual hallucinations, perceptual distortion of colors, shapes and light intensity, decrease in contour integration and surround suppression. Other, frequently present eye disorders include impaired visual acuity, strabismus and nystagmus.

Conclusion: Visual impairments are one of the most important features of schizophrenia, which could help in defining the disease state and assigning appropriate treatment.

Key words: schizophrenia - visual impairments - retina - biomarkers

\section{INTRODUCTION}

Schizophrenia is a multifactorial neurodevelopmental disorder associated with cognitive dysfunction and changes in primary sensory processing (Insel 2010, Graticelli et al. 2015). The disease itself has several main characteristics, which are classified into positive symptoms such as hallucinations, delusions, disorganized speech and behavior, and negative symptoms such as apathy, social withdrawal, emotional flattening and avolition (Nasrallah et al. 2011). While it is currently still being defined according to the above-mentioned features, schizophrenia remains a highly heterogeneous disorder. Simply stated, two different patients with an established diagnosis of schizophrenia could present with unique and seemingly unrelated signs and symp, probably reflecting different underlying psychopathological mechanisms and processes. Although our current research and clinical approaches are still mostly based on this "unifying" theory, the demand for stratification of the disease on many different levels has also been recognized (Hahn et al. 2017, Radic et al. 2018). Contrary to previous understandings, recent research has shown that schizophrenia is multisystemic rather than being a brain-centered disease (Pillinger et al. 2018). Numerous studies have shown that visual impairments are one of the most important features of schizophrenia, which could serve to define the disease state and thus appropriate treatment (Kogata \& Iidaka 2018). In the early stages of the disease, patients often display symptoms of visual deterioration, which can gradually increase other clinical symptoms and ultimately have an impact on their quality of life (Graticelli et al. 2015). The retina is an embryonic extension of the brain and may be involved in the pathophysiology of visual impairment in patients with schizophrenia (Silverstein \& Rosen 2015). Findings of ophthalmological studies have indicated that schizophrenic patients present different visual abnormalities in the initial components of the optic tract (Schonfeldt-Lecuona et al. 2016). There are many functional and structural deficits of the eye that could lead to disorders in visual processing and experiences that are exhibited in the disease. These include poor visual acuity, cataracts, strabismus, nystagmus, retinal venulae widening, various retinopathies and maculopathies, retinal nerve fiber layer (RNFL) thinning, dopamine and glutamate abnormalities in the retina and impaired color vision. 
Included also are reduced contrast sensitivity, visual hallucinations, decreased saccades and smooth pursuit eye movements (SPEMs), distortion of shapes and light intensity, abnormal electroretinogram (ERG) waveforms etc. Some of these features are attributed to the disease itself, while others could be related to other comorbidities or medication usage (Levy et al. 2010, Crook et al. 2014, Gonzales-Hernandez et al. 2014, Adams \& Nasrallah 2017, Torrey \& Yolken 2017). The connection between schizophrenia and the eye is even more interesting if we consider that it has never been documented that a congenitally blind person has schizophrenia (Silverstein et al. 2013). It is hypothesized that blindness serves as a protective mechanism through strengthening and maintenance of certain cognitive functions. Bearing in mind the above-described relation between the eye and schizophrenia, and the numerous questions the relationship raises, it is clear why the eyes are starting to be called ,the window to mental health". Within this review article, the authors present recent findings on the structural and functional abnormalities of the retina and related structures involved in visual processing, which could advance our knowledge and understanding of schizophrenia.

\section{METHODS}

To provide a literature review of visual impairments in schizophrenia, we performed a PubMed/MEDLINE and Scopus search to identify all articles on the topic up to the end of 2018. We used the following search terms: "schizophrenia AND visual impairments", "schizophrenia AND retinal abnormalities", "schizophrenia AND neurotransmitters", "schizophrenia AND abnormalities in eye movements", "schizophrenia AND visual processing", "schizophrenia AND perceptual disturbances" and "antipsychotic drugs AND ocular abnormalities". After the screening of all identified articles in English, only those that met the criteria for eligibility were included in the study. The reference list of the articles included in the review was manually checked for relevant studies.

\section{STRUCTURAL AND FUNCTIONAL CONSIDERATIONS OF THE RETINA}

The retina develops from the neuroectoderm, and it is the only part of the central nervous system that is exposed to direct analysis. The retina is considered as a "window to the brain" due to both being similar in structure and function (Jindal 2015, SchonfeldtLecuona et al. 2016). The axons of retinal ganglion cells (RGC), which form RNFL, leave the retina as the optic nerve and make synapses at the lateral geniculate nucleus (LGN), where they provide input to the magnocellular and parvocellular pathways. Observation of the retinal structure gives us insight into the functioning of both pathways, which are usually impaired in schizophrenia (Lalor et al. 2012, Jahshan et al. 2017). Multiple retinal functional and/or structural abnormalities are found in patients with schizophrenia. They may reflect the state of the brain structure and function and serve as a marker of brain tissue loss (Kemenyova et al. 2014). Moreover, retinal changes in schizophrenic patients may indicate structural and functional deficits, which exist throughout the brain and could be associated with the etiology of the disease. Visual abnormalities include reduced visual acuity, visual distortions, decreased contrast sensitivity, impaired color vision and eye tracking dysfunction (Gagne et al. 2015, Silverstein \& Rosen 2015). Retinal changes include microvascular, functionnal and structural abnormalities so that ophthalmological findings may provide valuable information in clarifying the pathogenesis of the disease. However, retinal abnormalities are not entirely particular to schizophrenia, as they can also be present in other neurodevelopmental psychiatric disorders. These findings could interfere with the presentation of the disease itself and also alter the perception of comorbidities and the side effects of medication. Retinal changes could help in better diagnostic and therapeutic approaches or serve as a predictive and preventive biomarker in the case of schizophrenia (Hosak et al. 2018). Recent studies have indicated that schizophrenia may be associated with retinal venulae widening. Thus, fundus examination and retinal vessels' documentation by fundus photography provide an evaluation of retinal microvasculature as an insight into cerebral vessels and could serve as a marker of disease vulnerability (Adams \& Nasrallah 2017, Hosak et al. 2018). Wider retinal venules in the patients with schizophrenia suggest a chronically insufficient supply of oxygen to the brain and could contribute to the appearance of psychotic symptoms in childhood and adulthood. In addition, wider venulae were found in children with temporary psychotic symptoms, which could be an essential marker for the subsequent development of psychosis (Meier et al. 2015). It was also shown that the widening of the retinal venule correlates significantly with childhood IQ and neurophysiological functioning during middle age (Shalev et al. 2013).

Due to known properties of the visual cortex, some aspects of functional visual impairment in schizophrenia involve deficient orientation discrimination. It seems that other aspects, such as reduced susceptibility to certain visual illusions, imply the reduced influence of stored knowledge on perception, which reflects damaged connections between the frontal, parietal and higher-level visual regions (Keane et al. 2013). The retina consists of more than 20 types of RGC, where the signals are integrated into LGN and each type of RGC contributes to a different aspect of the visual stimulus (Roska \& Meister 2014). Therefore, some visual processing damage may arise from the abnormal coordination of RGC signals into the LGN. Midget bipolar and 
ganglion cells (GC) project into the LGN parvocellular pathway and play a role in color processing, while parasol GC, which projects into the LGN magnocellular pathway, is essential for contrast sensitivity. Dysfunction of these cells could explain the impaired color vision and contrast sensitivity in schizophrenic patients (Crook et al. 2014). The retina, as part of the central nervous system, was established as being suitable for examination of psychiatric disorders using ERG (Hebert et al. 2017). Results from electrophysiological studies have demonstrated different ERG abnormalities, which indicate retinal signal processing dysfunction (Balogh et al. 2008, Hebert et al. 2015). ERG studies in schizophrenic patients have revealed abnormalities of a wave activity in the photoreceptors (both rod and cone) or $\mathrm{b}$ - wave activity in the bipolar and Muller cells (Warner et al. 1999, Hebert et al. 2015). It has been suggested that during acute psychosis there is an abnormal retinal function at the level of photoreceptors and bipolar and Muller cells. It is not yet clear if these findings are caused by later visual processing impairments or if they reflect parallel disturbances in brain function (Silverstein \& Rosen 2015). It has been considered that reduced a-wave amplitude in patients with schizophrenia arises due to the decreased level of essential fatty acids in the cell membrane of photoreceptors (Warner et al. 1999). There is also a notable negative relationship between a-wave amplitude and positive symptoms of the disease (Balogh 2008). Besides, rod retinal response is reduced in youths with high risk and in schizophrenic patients. Therefore, rod response anomaly may represent an early and specific biomarker before the onset of the disease and prove meaningful for further genetic and prevention research. It has been published that healthy children or adolescents at risk have decreased amplitude in rod b- and cone a-wave, which has also been found in adult patients with the full-blown disorder. In other words, ERG changes that occur many years before the onset of the disease could help clarify the underlying pathophysiological processes of schizophrenia (Hebert et al. 2015). A recent study, using portable flash electroretinography (fERG) confirmed the above-described findings, pertaining to reduced photoreceptor and bipolar cell functioning, and discovered that a portable device can detect the same anomalies and thereby opened further possibilities for more routine use of this screening method (Demmin et al. 2018)

Structural retinal changes in schizophrenia can be measured by optical coherence tomography (OCT), a non-invasive imaging technique, that can analyze retinal structure in vivo. Data of OCT scans are highly reliable and comparable to those of histologic examinations of retinal tissue (Vajzovic et al. 2012). Results from OCT studies on patients with schizophrenia have shown RNFL thinning and macular volume reduction. They have demonstrated that reduced macular volume was related to an increased expression of positive symptoms
(Chu et al. 2012), while RNFL thinning was related to disease chronicity (Lee et al. 2013; Schonfeldt-Lecuona et al. 2019). OCT studies have documented that RNFL, macular thickness, and macular volume are significantly decreased in the chronic phase of schizophrenia. The authors have underlined that OCT may provide useful findings that will help to control the progression of the disease (Hosak et al. 2018). Although most OCT studies among schizophrenic patients have shown a reduction of RNFL and macular thickness (Lee et al. 2013; Schonfeldt-Lecuona et al. 2019), Silverstein et al. did not confirm these findings (Celik et al. 2016, Yilmaz et al. 2016, Silverstein et al. 2017). In their studies, schizophrenic patients and controls did not differ significantly in RNFL or macula parameters, when age and medical comorbidity adjustments were made. Besides this, hypertension and diabetes, which often co-occur with schizophrenia and are commonly inadequately managed, can independently cause retinal thinning (Silverstein et al. 2017). It has also been proposed that acute psychosis is in correlation with inflammation and tissue swelling, which can disable the detection of retinal atrophy (Ascaso et al. 2015). Furthermore, the cup-to-disc ratio was enlarged and associated with cognitive symptoms in patients with schizophrenia, which seems to be mediated by RNFL thinning, loss of retinal capillaries and an enlarged optic nerve (Geyman et al. 2017). On the other hand, significant macular thinning predominantly occurred in the inner macula layers, suggesting that macular thinning may be a sign of degenerative, vascular and inflammatory processes that can co-occur in the brain itself (Ascaso et al. 2015, Joe et al. 2018). These parameters also correlated with the severity and progression of the disease (Satue et al. 2016).

Choroid, as the main source of the vascular supply of the outer retinal layers, recently came into focus as a possible biomarker for schizophrenic patients. Choroidal thinning was found mainly in those patients who are resistant to standard therapy and in patients with co-occurring diabetes who have no signs of diabetic retinopathy (Querques et al. 2012, Celik et al. 2016). Additionally, schizophrenia has been singled out as an independent risk factor for microvascular dysfunction (Lizano et al. 2016, Hua et al. 2017). Consequently, reduction of choroidal thickness could be a marker of different types of vascular dysfunction associated with schizophrenia (Joe et al. 2018). It has also been observed that RNFL thinning is associated with smoking in schizophrenic patients, although this thinning has not been found in "healthy smokers." This implicates that schizophrenia and smoking are not in a direct relationship (Patel et al. 2016, Duman et al. 2017). It is considered that disturbances of visual processing, which comprise color perception, contrast sensitivity, and reading, may develop because of RGC loss (Revheim et al. 2014). Because of that, RGC loss as the leading cause of RNFL thinning could be used as a biomarker for various retinal disorders. 


\section{THE ROLE OF NEUROTRANSMITTERS IN RETINAL FUNCTIONING}

The role of dopamine is well described in the literature considering the pathophysiology of schizophrenia. It has been established that hypodopaminergia in the mesocortical pathway is responsible for negative, while hyperdopaminergia in the mesolimbic pathway is responsible for positive symptoms in schizophrenic patients (Littrell \& Schneiderhan 1996). Dopamine is the most crucial neurotransmitter and modulator in the retina, which could explain the influence of schizophrenia on the visual system and plays a major role in the signaling of the retinal amacrine and interplexiform cells (Carlsson 1988). The main influence of dopamine on amacrine cells, which are primarily crucial for dark adaptation of the retina, is to uncouple electronic junctions that connect some of these cells. Dopamine participates in the physiological activities of RGC, so its dysfunction, which is present in schizophrenia, may modify visual processing by remodeling the capacity of the RGC receptive field. Animal studies have confirmed that dopaminergic deficiency in the retina causes a decrease in the number of retinal amacrine cells (Frederick et al. 1982). Reductions and excesses in dopamine activity in the brain also occur in the retina of schizophrenic patients (Silverstein \& Rosen 2015). Dopamine deficiency may reduce, while dopamine excess may increase color perception. In the acute onset of schizophrenia, dopamine values are increased, while those same values are normalized in phases of disease remission (Kegeles et al. 2010). Therefore, visual disturbances during acute psychotic episodes are opposite to those observed during antipsychotic treatment. Findings implicate that hyperdopaminergia is in correlation with excessive low spatial frequency processing and this is in accordance with excessive magnocellular responsivity and subjective visual changes that occur at the beginning of the disease (Keri \& Benedek 2007).

It has also been proposed that glutamate, another essential neurotransmitter in the retina, causes the destruction of RGC and plays a role in the process of neurodegeneration in schizophrenic patients (Sucher et al. 1997, Javit 2007). Schizophrenia is characterized by N-methyl-D-aspartate receptor (NMDAr) dysregulation and hypoactivity that leads to increased dopamine and glutamate release with consequent visual distortions and hallucinations. Accordingly, the use of NMDAr antagonists, like ketamine, may cause retinal hypoxia, cell death and reduced visual evocated potentials (VEPs) (Gonzales-Hernandez et al. 2014). Despite the present knowledge, the relationship between neurotransmitter changes and visual disturbances in schizophrenic patients has not yet been fully elucidated.

\section{ABNORMALITIES IN EYE MOVEMENTS}

Studies have revealed that the main pathological substrate underlying schizophrenia is structural brain damage (Keshavan et al. 2008). Although eye movements are affected in many neurodegenerative diseases, their abnormalities are one of the most reliable and reproducible impairments associated with schizophrenia (Fernandez et al. 2015, Coubard 2016, Dowiasch et al. 2016). Saccades and smooth pursuit eye movements (SPEMs) are two different types of oculomotor control, which act synergistically for visual tracking. Saccades are primarily directed toward stationary targets, while SPEMs are elicited to track moving targets. Studies have revealed a notable decrease in the control of saccades and SPEMs in schizophrenic patients (Levy et al. 2010, Benson et al. 2012). Results have shown that voluntary saccades are damaged, whereas reflexive saccades are preserved (Picard et al. 2012, Bender et al. 2013). Schizophrenic patients with mild neurological impairment have decreased saccadic adaptation and a substantial vergence tracking deficit (Bolding et al. 2014). Abnormal SPEMs suggest that reduced tracking may serve as a biological marker for schizophrenia (Morita et al. 2017). They are also present more than normal in schizophrenia risk groups and first-degree relatives, so it has been proposed that SPEMs have a genetic origin (Mitropoulou et al. 2011, Smyrnis et al. 2011, Roberts et al. 2013). Morita et al. analyzed eye movement measures such as free viewing, fixation stability, and smooth pursuit tests. They found that only three out of 75 known eye movement measures discriminate schizophrenic patients with more than $80 \%$ accuracy. They also revealed that there is a significant correlation between eye movements, clinical symptoms and the use of antipsychotic drugs (Morita et al. 2017). Scanpath and visual search impairments, such as decreased scanning length and fewer fixations, were also found in schizophrenic patients (Minassian et al. 2005, Nishiura et al. 2007). Early defects of visual processing may be associated with high order cognitive deficits such as attention and memory deficits (Butler \& Javitt 2005). Considering perception and social interaction, head movement is markedly reduced during activities such as speaking (Leask et al. 2012). Thus, alerted head movements may reflect on the eye movements and cause abnormal eye-head coordination (Schwab et al. 2013). Schizophrenic patients showed a longer mean duration of fixation, reduced eye fixations and shorter mean scanning length. It has been published that a decreased responsive search score (RSS) and greymatter loss have been found in individuals with schizophrenia. RSS decline is associated with a reduction of grey-matter density in occipito-temporo-frontal brain parts, which underlie visual information and eye movement processing (Qui et al. 2011). Thus, RSS may be used as a reliable biomarker to predict the severity of hallucinations in schizophrenic patients (Qui at al 2018). Moreover, the brain structural abnormalities correlate strongly with cognitive function deficits and positive psychotic symptoms (Liao et al. 2015, Mottahedin et al. 2017). 


\section{VISUAL PROCESSING AND PERCEPTUAL DISTURBANCES}

Visual sensory impairments and concomitant functional deficits are well described in schizophrenia as they occur because of structural and functional damage of RGC, magnocellular pathway neurons and parvocellular pathway neurons (Jahshan et al. 2017). It is considered that neural connections and synapses are affected in the visual cortex of schizophrenic patients. Impairments in visual perception have been depicted as resulting in experiencing the world in fragment parts as opposed to the continuous streaming of visual information (Anderson et al. 2017). Studies have confirmed that disrupted visual processing of the magnocellular pathway may result in the decrease of sensory processing, orientation discrimination, visual integration, trajectory and spatial localization, backward masking, motion tracking and neurophysiological responses to visual stimuli (Butler et al. 2005, Yeap et al. 2008, Khosravani \& Goodarzi 2013). Dysfunction in the magnocellular pathway is especially presented with reduced contrast sensitivity (Yeap et al. 2008), besides it may contribute to cognitive deficits in attention, working memory and executive functioning (Martinez et al. 2008). Although the test of contrast sensitivity is considered the best way to explore magnocellular integrity, schizophrenic patients exhibit uniform reductions in contrast sensitivity, which may be related to attention disorders or the effects of drugs (Skottun \& Skoyles 2007).

Visual perceptual abnormalities (VPAs) are present in more than $60 \%$ of schizophrenic patients. Visual distortions are associated with a worsening of negative symptoms in remitted patients and an increased psychosis risk in prodromal patients (Keri et al. 2005). These symptoms usually occur in the chronic phase of the disease and some visual deficits decline with its duration, such as contrast sensitivity, contour integration and surround suppression (Keane et al. 2016). Schizophrenic patients report more VPAs than patients with other psychotic disorders. VPAs are associated with more positive symptoms, earlier age of the onset of the disease and lower premorbid functioning (Keane et al. 2018). Authors have investigated the clinical importance of VPAs and found that the duration of the disease had less notability for the occurrence of VPAs. Their findings of more abnormal perception of faces, bodies and objects' motion are consistent with previous reports of abnormal visual behavioral performance and brain activation (Bauser et al. 2012). However, in contrast to this, another study showed that VPAs are in correlation with negative but not positive symptoms (Kery et al. 2005). Electrophysiological studies with VEP revealed deficits in the perceptual organization and visual processing (Butler \& Javitt 2005, Silverstein \& Keane 2011). The early stage of schizophrenia is characterized by visual changes such as a perceptual distortion of colors, shapes and light intensity (Torrey \& Yolken 2017). Schizophrenic patients also tended to have visual hallucinations in $73 \%$ of cases and the use of antipsychotic drugs had a positive effect on this (Phillipson \& Harris 1985). The intensity of hallucinations is significantly associated with volumetric changes of brain structures including grey matter in frontal, parietal and temporal regions as well as in the paralimbic system (Padmanabhan et al. 2015, Qui et al. 2018). Because of ocular misalignment, amblyopia and difficulties in depth perception may occur. Schizophrenic patients perform worse on visual tasks such as contour integration (Silverstein et al. 2012). In the literature, there is no evidence of schizophrenia occurrence in cases of congenital blindness, suggesting that blindness has a protective role. However, congenital blindness does not have a protective role in other psychiatric conditions (Silverstein et al. 2013). Frequently present eye disorders in schizophrenic patients are impaired visual acuity, strabismus, and nystagmus. It has been detailed that patients with schizophrenia had decreased visual acuity for distance and near vision, limiting their activities of daily living and social functioning (Vierto et al. 2012). Children with impaired visual acuity at age four had markedly increased the incidence of schizophrenia as adults (Hassan et al. 2015). Patients with schizophrenia also have strabismus more often than the general population. It has been found that strabismus in the form of exotropia was present in $13 \%$ of schizophrenic patients (Yoshitsugu et al. 2006).

\section{USE OF ANTIPSYCHOTIC DRUGS AND OCULAR ABNORMALITIES}

Results of numerous studies have shown that the use of antipsychotic drugs may contribute to visual impairment (Viertio et al. 2007). Antipsychotic drugs cause pupilar dilatation and blurred vision due to their anticholinergic action. As a serotonin agonist, some types of antipsychotics for schizophrenia treatment may increase intraocular pressure and lead to glaucoma (Souza et al. 2008). Studies have reported that patients with schizophrenia had lens opacities, cataract, and corneal pigmentation. Cataract was mostly present in patients treated with the first generation of antipsychotic drugs (Smith et al. 1997). The derivate of phenothiazine can cause pigment deposition on the corneal endothelium, conjunctiva and anterior lens capsule. Antipsychotic drugs blockade retinal dopamine receptors, which may cause the death of RGC resulting in the thinning of retinal layers (Silverstein \& Rosen 2015). Phenothiazine has a toxic activity on the photoreceptors with consequent damage and atrophy of retinal pigment epithelium, manifesting as blurred vision, scotomas, dyschromatopsia, and nyctalopia. Use of thioridazine may cause pigmentary retinopathy characterized with reduced visual acuity, retinal pigment deposits, and suppression of dark-adapted ERG responses (Adams \& Nasrallah 2017). Therefore, retinal monitoring is vital at the beginning of therapy and regular ophthalmologic control exams are necessary to detect any retinotoxic effects of 
antipsychotic drugs (Andreoli et al. 2018). Although psychotropic drugs may have an influence on eye movements, it is considered that oculomotor deficits in schizophrenic patients are primarily the result of the disease itself (Anthony 2018). However, use of benzodiazepines has some effects on ocular motility such as smooth pursuit tracking deficits, decreased saccadic peak velocity and vestibular ocular reflex gain. Lencer et al. reported that there was no notable correlation between smooth pursuit measurements and antipsychotic usage. However, some authors reported an increase of smooth pursuit abnormalities both after the beginning and prolonged treatment with antipsychotic drugs (Reilly et al. 2008, Lencer et al. 2015). Schizophrenic patients treated by dopamine receptor agonists showed visual acuity deterioration, problems in contrast sensitivity and longer VEP latency (Kelemen et al. 2013). Antipsychotic drugs commonly did not affect visual acuity, but phenothiazines could impair near vision through the effect on the ciliary muscle during accommodation.

\section{ENDOPHENOTYPES AND FUTURE DIRECTIONS}

Another important aspect of this review are endophenotypes. Endophenotypes as such represent laboratory-based measures that show deficits in schizophrenia patients and to a lesser extent deficit in their first degree "unaffected" relatives (Braff \& Tamminga 2016). Eye movement impairment have been recognized in schizophrenia patients for more than hundred years and were considered as an endophenotype in general (Obyedkov et al. 2019) while now distinct eye movement tasks such as smooth pursuit eye movement (SPEM) disturbance, antisaccade (AS) and memory-guided saccade (MGS) are considered even more specific endophenotypes (Park et al. 2009; Caldani et al. 2016). SPEM has been proven to have a high heritability and accuracy with schizophrenia patients (Kikuchi et al. 2018) and although there is no correlation between SPEM abnormality and six COMT gene polymorphisms (Park et al. 2009), 17 SNPs susceptibility loci have been identified for SPEM in schizophrenia patients in an effort to elucidate the genetic association of eye movements from a different perspective in the smooth pursuit test (Kikuchi et al. 2018). Increased rate of errors in tasks pertaining to saccade eye movement are one of the most replicable findings in schizophrenia research and have been linked with poor inhibition control as well as agitation and excitement (Obyedkov et al. 2019). On top of that, recent finding in ERG and OCT (Demmin et al. 2018; Schonfeldt-Lecuona et al. 2019) further advocate the role of the eye, and eye movements as such, as a viable source for endophenotypes in schizophrenia. It is evident why endophenotypes are considered ideally compatible with concepts such as the Research Domain Criteria and thus "bridge genomic complexity and disorder heterogeneity." Furthermore, recent advances in understanding underlying epigenetic mechanisms in schizophrenia (Jakovljevic \& Borovecki 2018) make the endophenotypes, relating to the eye, an even more interesting source of information as the eye is easy to research and track the changes in shorter intervals. The combination of endophenotypes research and epigenetic profiling could be used to identify certain diseases subtypes and help to administer adequate medication accordingly. Considering the therapeutic aspects of schizophrenia, one should be aware of the complexity of human beings. Unique and specific features of the disease highlight the importance of person-centered care (Jakovljević 2015). In other words, such care should be personalized, integrative and creative, considering different visual impairments, treatment modalities and patients' wellbeing (Jakovljević 2015, Jurišić et al. 2018).

\section{CONCLUSION}

Visual impairments are one of the most important features of schizophrenia, which could serve to define the state of the disease and therefore aid with the administering of appropriate treatment. The most common visual disturbances in schizophrenic patients include abnormalities in retinal structure and function, eye positions and movements, visual processing, visual perception and visual acuity. Moreover, retinal changes in these patients may reflect structural and functional deficits of the brain and serve as a marker of brain tissue loss. The notion that visual impairments, or even compromised multisensory integration, is a risk factor for schizophrenia is even more prominent in the context of undocumented schizophrenia in congenitally blind people. Different parameters derived from fundus examination, ERG and OCT seem to be able to discriminate healthy subjects from high-risk individuals or ones who are burdened by schizophrenia. These methods provide valuable diagnostic and prognostic stratification with high practical potential by enabling insight into vascular, inflammation and degenerative status of the retina, but also brain itself (as the retina is an embryonic extension of the brain, and it is the only part that can be directly examined). Although all these findings need to be replicated by more extensive research, it is evident that the intricate relationship between visual impairments and schizophrenia could provide new insights into the etiology and pathophysiology of the disease, as endophenotypes. These findings could also enable more reliable signs of disease staging and progression as well as clarify distinct questions that are still unanswered in schizophrenia.

Finally, these findings currently provide a useful aid in answering the question, with limited possibilities to answer why, when and how questions. In other words, the question on what could be the cause and what the consequence (of the disease itself, co-occurring condition, and/or treatment) remains. This dichotomy might be of special relevance here, as recently, the classical theory of visual processing has been questioned. Within the prediction and error processing (PEP) paradigm, it 
has been recognized that retina does not serve as a purely passive "converter" of sensory signals which are later integrated in constructs representations in visual cortex (Clark, 2015, Summerfield et al. 2006). In other words, when sensory signal reaches retina cells they are confronted with certain "predictions" embedded in retina and only signals that are dissonant with those predictions are the ones that are transduced to a higher level brain regions (which than reduces dissonance either by refining predictions embedded in retina or by modifying sensory inputs) (Clark 2015, Summerfield et al. 2006). So, as perception cannot be separated from prediction, examining retina could possibly provide insight in erroneous inferences brain burdened with illness makes in the environment filled with ambiguous signals.

In order to move forward, we believe there is a great need for "vertical" and "horizontal" integration of knowledge, where ophthalmology and its respected subjects should be able to provide a valuable "piece of puzzle" for establishing a comprehensive theory of schizophrenia-related disorders that would be able to emphasize clinically quite evident each individual patient's uniqueness.

\section{Acknowledgements: None.}

\section{Conflict of interest: None to declare.}

\section{Contribution of individual authors:}

Study conception and design: Darija Jurišić, Ivan Ćavar \& Marko Ćurković;

Acquisition, analysis and interpretation of data: Darija Jurišić, Ivan Ćavar, Irena Sesar, Antonio Sesar, Jakša Vukojević \& Marko Ćurković;

Writing of the manuscript: Darija Jurišić, Ivan Ćavar, Irena Sesar, Antonio Sesar, Jakša Vukojević \& Marko Ćurković.

\section{References}

1. Adams SA \& Nasrallah HA: Multiple retinal anomalies in schizophrenia. Schizophr Res 2018; 195:3-12

2. Anderson E, Tibber M, Schwarzkopf DS, Shergill SS, Fernandez-Egea E, Rees $G$ et al.: Visual population receptive fields in people with schizophrenia have reduced inhibitory surrounds. J Neurosci 2017; 67:1546-56

3. Andreoli MT, Mittra RA, Mieler WF: Drug toxicity of the posterior segment. In: Schachat AP, Sadda SR, eds. Ryan's Retina, 6th ed. New York: Elsevier 2018; pp. 1719-45

4. Anthony SA: Focus on eye care in schizophrenia. Clin Exp Optom 2018. doi: 10.1111/cxo.12826. [Epub ahead of print]

5. Ascaso FJ, Rodriguez-Jimenez R, Cabezon L, Lopez-Anton $R$, Santabarbara J, De La Camara C et al.: Retinal nerve fiber layer and macular thickness in patients with schizophrenia: influence of recent illness episodes. Psychiatry Research 2015; 229:230-6
6. Balogh Z, Benedek G, Kéri S: Retinal dysfunctions in schizophrenia. Prog Neuropsychopharmacol Biol Psychiatry 2008; 32:297-300

7. Bauser DS, Thoma P, Aizenberg V, Brüne M, Juckel G, Daum I: Face and body perception in schizophrenia: A configural processing deficit? Psychiatry Res 2012; 195:9-17

8. Bender J, Reuter B, Möllers D, Kaufmann C, Gallinat J, Kathmann N: Neural correlates of impaired volitional action control in schizophrenia patients. Psychophysiology 2013; 50:872-84

9. Benson PJ, Beedie SA, Shephard E, Giegling I, Rujescu D, St Clair D: Simple viewing tests can detect eye movement abnormalities that distinguish schizophrenia cases from controls with exceptional accuracy. Biological Psychiatry 2012; 72:716-24

10. Bolding MS, Lahti AC, White D, Moore C, Gurler D, Gawne TJ et al: Vergence eye movements in patients with schizophrenia. Vis Res 2014; 102:64-70

11. Braff DL \& Tamminga CA: Endophenotypes, Epigenetics, Polygenicity and More: Irv Gottesman's Dynamic Legacy. Schizophr Bull 2017; 43:10-16. doi: 10.1093/schbul/sbw157

12. Butler PD \& Javitt DC: Early-stage visual processing deficits in schizophrenia. Current Opinion in Psychiatry 2005; 18:151-7

13. Carlsson A: The current status of the dopamine hypothesis of schizophrenia. Neuropsychopharmacol: Off Publ Am Coll Neuropsychopharmacol. 1988; 1:179-86

14. Caldani S, Bucci MP, Lamy JC, Seassau M, Bendjemaa N, Gadel R, Gaillard R, Krebs MO, Amado I: Saccadic eye movements as markers of schizophrenia spectrum: Exploration in at-risk mental states. Schizophr Res. 2017; 181:30-37. doi: 10.1016/j.schres.2016.09.003

15. Celik M, Kalenderoglu A, Sevgi Karadag A, Bekir Egilmez $O$, Han-Almis B, Simsek A: Decreases in ganglion cell layer and inner plexiform layer volumes correlate better with disease severity in schizophrenia patients than retinal nerve fiber layer thickness: findings from spectral optic coherence tomography. European Psychiatry 2016; 32:9-15

16. Chu EM, Kolappan M, Barnes TR, Joyce EM, Ron MA: A window into the brain: an in vivo study of the retina in schizophrenia using optical coherence tomography. Psychiatry Research 2012; 203:89-94

17. Clark A. Surfing Uncertainty: Prediction, Action and the Embodied Mind. 1st ed. New York: Oxford University Press, 2015

18. Coubard OA: An integrative model for the neural mechanism of eye movement desensitization and reprocessing (EMDR). Front Behav Neurosci 2016; 10:52

19. Crook JD, Packer OS, Dacey DM: A synaptic signature for $\mathrm{ON}$ - and OFF-center parasol ganglion cells of the primate retina. Vis Neurosci 2014; 31:57-84

20. Demmin DL, Davis Q, Roché M, Silverstein SM: Electroretinographic anomalies in schizophrenia. J Abnorm Psychol. 2018; 127:417-428. doi: 10.1037/abn0000347. PubMed PMID: 29745706

21. Dowiasch S, Backasch B, Einhauser W, Leube D, Kircher T, Bremmer F: Eye movements of patients with schizophrenia in a natural environment. Eur Arch Psychiatry Clin Neurosci 2016; 266:43-54

22. Duman R, Duman R, Sabaner MC, Cetinkaya E: Effect of smoking on the thickness of retinal layers in healthy smokers. Cutaneous and Ocular Toxicology 2017; $36: 366-9$ 
23. Fernandez G, Castro LR, Schumacher M, Agamennoni OE: Diagnosis of mild Alzheimer disease through the analysis of eye movements during reading. $J$ Integr Neurosci 2015; 14:121-33

24. Frederick JM, Rayborn ME, Laties AM, Lam DM, Hollyfield JG: Dopaminergic neurons in the human retina. J Comp Neurol 1982; 210:65-79

25. Gagné AM, Lavoie J, Hébert M, Maziade M: Revisiting visual dysfunctions in schizophrenia from the retina to the cortical cells: a manifestation of aberrant neurodevelopment. Prog. Neuro-Psychopharmacol. Biol Psychiatry $2015 ; 62: 29-34$

26. Geyman LS, Garg RA, Suwan Y, Trivedi V, Krawitz BD, Mo $S$ et al: Peripapillary perfused capillary density in primary open-angle glaucoma across disease stage: an optical coherence tomography angiography study. British Journal of Ophthalmology 2017; 101:1261-8

27. Gonzalez-Hernandez JA, Pita-Alcorta C, Padron A, Finale A, Galan L, Martinez E et al: Basic visual dysfunction allows classification of patients with schizophrenia with exceptional accuracy. Schizophr Res 2014; 159:226-33

28. Graticelli CPB, Abe RY, Diniz-Filho A, Benites Vaz-deLima F, Paranhos Jr. A, Medeiros FA: Ophthalmology issues in schizophrenia. Curr Psychiatry Rep 2015; 17:28

29. Hahn T, Nierenberg AA, Whitfield-Gabrieli S: Predictive analytics in mental health: applications, guidelines, challenges and perspectives. Mol Psychiatry 2017; 22:37-43

30. Hassan MB, Hodge DO, Mohney BG: Prevalence of mental health illness among patients with adult-onset strabismus. Strabismus 2015; 23:105-110

31. Hébert M, Mérette C, Paccalet T, Émond C, Gagné AM, Sasseville A et al: Light evoked potentials measured by electroretinogram may tap into the neurodevelopmental roots of schizophrenia. Schizophr Res 2015; 162:294-5

32. Hébert M, Mérette C, Paccalet T, Gagné AM, Maziade M: Electroretinographic anomalies in medicated and drug free patients with major depression: Tagging the developmental roots of major psychiatric disorders. Progress in Neuro-Psychopharmacology \& Biological Psychiatry 2017; 75:10-15

33. Hosak L, Sery O, Sadykov E, Studnicka J: Retinal abnormatilites as a diagnostic or prognostic marker of schizophrenia. Biomed Pap Med Fac Univ Palacky Olomouc Czech Repub 2018; 162:159-64

34. Hua J, Brand AS, Lee S, Blair NIS, Wu Y, Lui $S$ et al: Abnormal grey matter arteriolar cerebral blood volume in schizophrenia measured with $3 D$ inflow-based vascularspace-occupancy MRI at 7T. Schizophr Bull 2017; 43:620-32

35. Insel TR: Rethinking schizophrenia. Nature 2010; 468:187-93

36. Jahshan C, Wolf M, Karbi Y, Shamir E, Rassovsky Y: Probing the magnocellular and parvocellular visual pathways in facial emotion perception in schizophrenia. Psychiatry Res 2017; 253:38-42

37. Jakovljevic M: Person-centred psychopharmacotherapy: what is it? Each patient is a unique, responsive and responsible subject. Psychiatr Danub 2015; 27(Suppl 1). S28-33

38. Jakovljevic $M$ \& Borovecki F: Epigenetics, Resilience, Comorbidity and Treatment Outcome. Psychiatr Danub 2018; 30:242-253. doi: 10.24869/psyd.2018.242

39. Javitt DC: Glutamate and schizophrenia: phencyclidine $N$-methyl-D-aspartate receptors, and dopamine-glutamate interactions. Int Rev Neurobiol. 2007; 78:69-108
40. Jindal $V$ : Interconnection between brain and retinal neurodegenerations. Molecular Neurobiology 2015; 51:885-92

41. Joe P, Ahmad M, Riley G, Weissman J, Smith TR, Malaspina D: A pilot study assessing retinal pathology in psychosis using optical coherence tomography: Choroidal and macular thickness. Psychiatry Research 2018; 263:158-61

42. Jurišić D, Sesar I, Ćavar I, Sesar A, Živković M, Ćurković M: Hallucinatory experiences in visually impaired individuals: Charles Bonnet syndrome - implications for research and clinical practice. Psychiatr Danub 2018; 30:122-8

43. Keane BP, Cruz LN, Paterno D, Silverstein SM: Selfreported visual perceptual abnormalities are strongly associated with core clinical features in psychotic disorders. Front Psychiatry 2018; 9:69

44. Keane BP, Paterno D, Kastner S, Silverstein SM: Visual integration dysfunction in schizophrenia arises by the first psychotic episode and worsens with illness duration. $J$ Abnorm Psychol 2016; 125:543-9

45. Keane BP, Silverstein SM, Wang Y, Papathomas TV: Reduced depth inversion illusions in schizophrenia are statespecific and occur for multiple object types and viewing conditions. J Abnorm Psychol 2013; 122:506-12

46. Kegeles LS, Abi-Dargham A, Frankle WG, Gil R, Cooper $T B$, Slifstein $M$ et al: Increased synaptic dopamine function in associative regions of the striatum in schizophrenia. Arch Gen Psychiatry 2010; 67:231-9

47. Kelemen O, Kiss I, Benedek G, Keri S: Perceptual and cognitive effects of antipsychotics in first-episode schizophrenia: the potential impact of GABA concentration in the visual cortex. Prog. Neuropsychopharmacol. Biol Psychiatry 2013; 47:13-9

48. Kemenyova $P$, Turcani $P$, Sutovsky S, Waczulikova I: Optical coherence tomography and its use in optical neuritis and multiple sclerosis. Bratisl Lek Listy 2014; 115:723-29

49. Keri $S$ \& Benedek G: Visual contrast sensitivity alterations in inferred magnocellular pathways and anomalous perceptual experiences in people at high-risk for psychosis. Vis Neurosci 2007; 24:183-9

50. Keri S, Kiss I, Kelemen O, Benedek G, Janka Z: Anomalous visual experiences, negative symptoms, perceptual organization and the magnocellular pathway in schizophrenia: a shared construct? Psychol Med 2005; 35:1445-11

51. Keshavan MS, Tandon R, Boutros NN, Nasrallah HA: Schizophrenia, "just the facts": what we know in 2008. part 3: neurobiology. Schizophr Res 2008; 106:89-107

52. Khosravani $N$ \& Goodarzi M: Patients with schizophrenia show deficits on spatial frequency doubling. Vis Res 2013; 93:49-53

53. Kikuchi M, Miura K, Morita K, Yamamori H, Fujimoto M, Ikeda $M$ et al: Genome-wide Association Analysis of Eye Movement Dysfunction in Schizophrenia. Sci Rep 2018; 8:12347. doi: 10.1038/s41598-018-30646-9

54. Kogata $T$ \& Iidaka $T$ : A review of impaired visual processing and the daily visual world in patients with schizophrenia. Nagoya J Med Sci 2018; 80:317-28

55. Lalor EC, De Sanctis P, Krakowski MI, Foxe JJ: Visual sensory processing deficits in schizophrenia: is there anything to the magnocellular account? Schizophrenia Research 2012; 139:246-52 
56. Leask SJ, Park B, Khana P, DiMambro B: Head movements during conversational speech in patients with schizophrenia. Therapeutic Advances in Psychopharmacology 2013; 3:29-31

57. Lee WW, Tajunisah I, Sharmilla K, Peyman M, Subrayan $V$ : Retinal nerve fiber layer structure abnormalities in schizophrenia and its relationship to disease state: evidence from optical coherence tomography. Investigative Ophthalmology and Visual Science 2013; 54:7785-92

58. Lencer R, Sprenger A, Reilly JL, McDowell JE, Rubin LH, Badner JA et al: Pursuit eye movements as an intermediate phenotype across psychotic disorders: Evidence from the B-SNIP study. Schizophr Res 2015; 169:326-33

59. Levy DL, Sereno AB, Gooding DC, O'Driscoll GA: Eye tracking dysfunction in schizophrenia: characterization and pathophysiology. Curr Top Behav Neurosci 2010; 4:311-47

60. Liao J, Yan H, Liu Q, Yan J, Zhang L, Jiang S et al: Reduced paralimbic system gray matter volume in schizophrenia: correlations with clinical variables, symptomatology and cognitive function. J Psychiatr Res 2015; 65:80-6

61. Littrell RA \& Schneiderhan M: The neurobiology of schizophrenia. Pharmacotherapy 1996; $16(6$ Pt 2):143S$147 S$ (discussion 166S-168S)

62. Lizano PI, Kashavan MS, Tandor N, Mathew IT, Mothi SS, Montrose DM et al: Angiogenic and immune signatures in plasma of young relatives at familiar high-risk for psychosis and first-episodes patients: a preliminary study. Schizophr Res 2016; 170:115-22

63. Martinez A, Hillyard SA, Dias EC, Hagler DJ Jr, Butler $P D$, Guilfoyle DN et al: Magnocellular pathway impairment in schizophrenia: evidence from functional magnetic resonance imaging. J Neurosci 2008; 28:7492-500

64. Meier MH, Gillespie NA, Hansell NK, Hewitt AW, Hickie $I B, \quad L u \quad Y$ et al: Retinal microvessels reflect familial vulnerability to psychotic symptoms: A comparison of twins discordant for psychotic symptoms and controls. Schizophr Res 2015; 164:47-52. doi: 10.1016/j.schres.2015.01.045

65. Minassian A, Granholm E, Verney S, Perry W: Visual scanning deficits in schizophrenia and their relationship to executive functioning impairment. Schizophrenia Research 2005; 74:69-79

66. Mitropoulou V, Friedman L, Zegarelli G, Wajnberg S, Meshberg J, Silverman JM et al: Eye tracking performance and the boundaries of the schizophrenia spectrum. Psychiatry Research 2011; 186:18-22

67. Morita K, Miura K, Fujimoto M, Yamamori H, YasudaY, Iwase $M$ et al: Eye movement as a biomarker of schizophrenia: using an integrated eye movement score. Psychiatry and Clinical Neurosciences 2017; 71:104-14

68. Mottahedin A, Ardalan M, Chumak T, Riebe I, Ek J, Mallard C: Effect of neuroinflammation on synaptic organization and function in the developing brain: implications for neurodevelopmental and neurodegenerative disorders. Front Cell Neurosci 2017; 11:190

69. Nasrallah HA, Tandon R, Keshavan M: Beyond the facts in schizophrenia: closing the gaps in diagnosis, pathophysiology and treatment. Epidemiol Psychiatr Sci 2011, 20:317-27

70. Nishiura S, Morita K, Kurakake K, Igimi H, Maeda H: Characteristics of left and right scanning in schizophrenia patients using exploratory eye movements: comparison with healthy subjects. Psychiatry and Clinical Neurosciences 2007; 61:487-94
71. Obyedkov I, Skuhareuskaya M, Skugarevsky O, Obyedkov V, Buslauski P, Skuhareuskaya T, Waszkiewicz N: Saccadic eye movements in different dimensions of schizophrenia and in clinical high-risk state for psychosis. BMC Psychiatry 2019; 19:110. doi: 10.1186/s12888-019-2093-8

72. Padmanabhan JL, Tandon N, Haller CS, Mathew IT, Eack $S M$, Clementz Baet al: Correlations between brain structure and symptom dimensions of psychosis in schizophrenia, schizoaffective, and psychotic bipolar I disorders. Schizophr Bull 2015; 41:154-62

73. Park BL, Shin HD, Cheong HS, Park CS, Sohn JW, Kim $B J$ et al: Association analysis of COMT polymorphisms with schizophrenia and smooth pursuit eye movement abnormality. J Hum Genet 2009; 54:709-12. doi: 10.1038/jhg.2009.102

74. Patel PJ, Foster PJ, Grossi CM, Keane PA, Ko F, Lotery $A$ et al: Spectral-Domain optical coherence tomography imaging in 67321 adults: associations with macular thickness in the UK biobank study. Ophthalmology 2016; 123:829-40

75. Phillipson OT \& Harris JP: Perceptual changes in schizophrenia: a questionnaire survey. Psycholog Med 1985; 15:859-66

76. Picard H, Le Seac'h A, Amado I, Gaillard R, Krebs MO, Beauvillain C: Impaired saccadic adaptation in schizophrenic patients with high neurological soft sign scores. Psychiatr Res 2012; 199:12-8

77. Pillinger T, D'Ambrosio E, McCutcheon R, Howes OD: Is psychosis a multisystem disorder? A meta-review of central nervous system, immune, cardiometabolic, and endocrine alterations in first-episode psychosis and perspective on potential models. Mol Psychiatry 2018; doi: 10.1038/s41380-018-0058-9 [Epub ahead of print]

78. Qiu L, Tian L, Pan C, Zhu R, Liu Q, Yan J et al: Neuroanatomical circuitry associated with exploratory eye movement in schizophrenia: a voxel-based morphometric study. PloS One 2011; 6:e25805

79. Qiu L, Yan H, Zhu R, Yan J, Yuan H, Han Y et al: Correlations between exploratory eye movement, hallucination, and cortical gray matter volume in people with schizophrenia. BMC Psychiatry 2018; 18:226

80. Querques G, Lattanzio R, Querques I, Del Turco C, Forte $R$, Pierro I et al: Enhanced depth imaging optical coherence tomography in type 2 diabetes. Invest Ophthalmol Vis Sci 2012; 53:6017-24

81. Radic K, Curkovic M, Bagaric D, Vilibic M, Tomic A, Zivkovic M: Ethical Approach to Prevention of Schizophrenia - Concepts and Challenges. Psychiatr Danub 2018; 30:35-40

82. Reilly JL, Lencer R, Bishop JR, Keedy S, Sweeney JA: Pharmacological treatment effects on eye movement control. Brain Cogn 2008; 68:415-35

83. Revheim N, Corcoran CM, Dias E, Hellmann E, Martinez A, Butler PD et al: Reading deficits in schizophrenia and individuals at high clinical risk: relationship to sensory function, course of illness, and psychosocial outcome. Am J Psychiatry 2014; 171:949-59

84. Roska B \& Meister M: The retina dissects the visual scene into distinct features. In: Chalupa L,Werner JS (Eds.), The new visual neurosciences. MIT Press, Cambridge, MA 2014

85. Roberts EO, Proudlock FA, Martin K, Reveley MA, AlUzri M, Gottlob I: Reading in schizophrenic subjects and their nonsymptomatic first-degree relatives. Schizophrenia Bulletin 2013; 39:896-907 
86. Satue M, Obis J, Rodrigo MJ, Otin S, Fuertes MI, Vilades E et al: Optical coherence tomography as a biomarker for diagnosis progression and prognosis of neurodegenerative diseases. J Ophthalmol 2016; 2016:e8503859

87. Schonfeldt-Lecuona C, Kregel T, Schmidt A, Pinkhardt EH, Lauda F, Kassubek J: From imaging the brain to imaging the retina: optical coherence tomography (OCT) in schizophrenia. Schizophrenia Bulletin 2016; 42:9-14

88. Schönfeldt-Lecuona C, Kregel T, Schmidt A, Kassubek J, Dreyhaupt J, Freudenmann RW et al: Retinal single-layer analysis with optical coherence tomography (OCT) in schizophrenia spectrum disorder. Schizophr Res. 2019. doi: 10.1016/j.schres.2019.03.022

89. Schwab S, Würmle O, Razavi N, Müri RM, Altorfer A: Eye-head coordination abnormalities in schizophrenia. PLoS One 2013; 8:e74845

90. Shalev I, Moffitt TE, Wong TY, Meier MH, Houts RM, Ding J: Retinal vessel caliber and lifelong neuropsychological functioning: retinal imaging as an investigative tool for cognitive epidemiology. Psychol Sci 2013; 24:1198-207

91. Silverstein SM \& Keane BP: Perceptual organization impairment in schizophrenia and associated brain mechanisms: review of research from 2005 to 2010. Schizophr Bull 2011; 37:690-9

92. Silverstein SM \& Rosen R: Schizophrenia and the eye. Schizophrenia Research: Cognition 2. 2015; 46-55

93. Silverstein SM, Paterno D, Cherneski L, Green S: Optical coherence tomography indices of structural retinal pathology in schizophrenia. Psychological Medicine 2017. https://doi.org/10.1017/S0033291717003555

94. Silverstein SM, Wang Y, Keane BP: Cognitive and neuroplasticity mechanisms by which congenital or early blindness may confer a protective effect against schizophrenia. Front Psychol 2013; 21:624

95. Silverstein SM, Wang $Y$, Roche MW: Base rates, blindness, and schizophrenia. Front Psychol 2013; 4:157

96. Skottun BC \& Skoyles JR: Contrast sensitivity and magnocellular functioning in schizophrenia. Vis Res 2007; 47:2923-33

97. Smith D, Pantelis C, Mcgrath J, Tangas C, Copolov D: Ocular abnormalities in chronic schizophrenia: clinical implications. Aust N Z J Psychiatr 1997; 31:252-6

98. Smyrnis N, Kattoulas E, Stefanis NC, Avramopoulos D, Stefanis CN, Evdokimidis I: Schizophrenia-related neure- gulin-1 single-nucleotide polymorphisms lead to deficient smooth eye pursuit in a large sample of young men. Schizophrenia Bulletin 2011; 37: 822-831

99. Souza VB, Moura Filho FJ, Souza FG, Pereira Filho SA, Coelho SS, Furtado FA et al: Intraocular pressure in schizophrenic patients treated with psychiatric medications. Arquivos Brasileiros de Oftalmologia 2008; 71:660-4

100. Sucher NJ, Lipton SA, Dreyer EB: Molecular basis of glutamate toxicity in retinal ganglion cells. Vis Res 1997; 37:3483-93

101. Summerfield $C$, Egner $T$, Greene $M$, Koechlin E, Mangels J, Hirsch J: Predictive Codes for Forthcoming Perception in the Frontal Cortex. Science 2006; 314:1311-14

102. Torrey EF \& Yolken RH: Schizophrenia and infections: the eyes have it. Schizophrenia Bulletin 2017; 43: 247-52

103. Vajzovic L, Hendrickson AE, O'Connell RV, Clark LA, Tran-Viet D, Possin D et al: Maturation of the human fovea: correlation of spectral-domain optical coherence tomography findings with histology. American Journal of Ophthalmology 2012; 154:779-89

104. Viertio S, Laitinen A, Perala J, Saarni SI, Koskinen S, Lonnqvist $J$ et al: Visual impairment in persons with psychotic disorder. Soc Psychiatr Epidemiol 2007; 42:902-8

105. Viertio S, Tuulio-Henrikson A, Perala J, Saarni SI, Koskinen $S$, Sihvonen $M$ et al: Activities of daily living, sotial functioning and their determinants in persons with psychotic disorder. European Psychiatry 2012; 27:409-15

106. Warner $R$ et al: Retinal function as a marker for cellmembrane omega-3 fatty acid depletion in schizophrenia: a pilot study. Biol Psychiatry 1999; 45:1138-42

107. Yeap S, Kelly SP, Sehatpour P, Magno E, Garavan H, Thakore JH et al: Visual sensory processing deficits in schizophrenia and their relationship to disease state. Eur Arch Psychiatry Clin Neurosci 2008; 258:305-16

108. Yilmaz U, Kucuk E, Ulgen A, Ozkose A, Demircan S, Ulusoy DM et al: Retinal nerve fiber layer and macular thickness measurement in patients with schizophrenia. European Journal of Ophthalmology 2016; 26:375-8

109. Yoshitsugu K, Yamada K, Toyota T, Aoki-Suzuki M, Minabe $Y$, Nakamura $K$ et al: A novel scale including strabismus and 'cuspidal ear' for distinguishing schizophrenia patients from controls using minor physical anomalies. Psychiatry Res 2006; 145:249-58

Correspondence:

Darija Jurišić, $M D, P h D$

Department of Ophthalmology, University Clinical Hospital Mostar

Bijeli Brijeg bb, 88 000, Mostar, Bosnia and Herzegovina

E-mail: jurisicd2@gmail.com 